\title{
Le travail "ni fait ni à faire": un risque pour la santé publique en France
}

Yves Clot $^{1}$

Il y a maintenant un lien établi entre dégradation de la santé au travail et dégradation de la santé publique. Ce lien affleure dans la conscience sociale et la vie de nos concitoyens. En France nous connaissons par exemple une crise des services d'urgence qui est le symptôme de la crise de l'Hôpital: le travail est devenu invivable car on ne s'y reconnaît plus, on y trahit son métier, ses techniques et ses valeurs, sa vocation. Mais maintenant la question déborde: aux urgences des hôpitaux il y a des morts faute de soin et il a fallu la crise du COVID 19 pour que les soignants reprennent exceptionnellement et pour un temps la main sur l'organisation de leur travail.

La question a pourtant été instruite de longue date. La qualité refoulée du travail est un poison social et subjectif, individuel et collectif. Les "règles de l'art" de l'activité des professionnels de première ligne, au bureau comme à l'usine, sont trop souvent ravalées par eux, sous l'effet de l'organisation du travail, pour que la santé publique reste protégée. L'analyse de l'activité empêchée et des sentiments d'impuissance qui lui sont associés dans la vie sociale du travail ne fait pas seulement partie des résultats classiques obtenus en psychologie du travail (CLOT, 2010). Les données statistiques l'attestent également en épaulant, de fait, nos résultats en clinique de l'activité dans de nombreux secteurs. Ainsi le service de recherche du Ministère du travail, la DARES, est-il parvenu à ces chiffres préoccupants: $35 \%$ des salariés de l'industrie et $37 \%$ dans la fonction publique d'Etat ou hospitalière déclarent ne pas ressentir (toujours ou souvent) "la fierté du travail bien fait" (COUTROT; DAVIE, 2014).

Plus grave, la parole de celles et ceux qui acceptent à reculons de faire un travail indéfendable à leurs propres yeux leur apparait de plus en plus inutile. Et là encore, ce n'est seulement la clinique du travail ordinaire qui le montre mais les données statistiques: toujours selon la DARES, la santé mentale des salariés s'améliore quand ils sont, non pas seulement consultés, mais partie prenante des décisions qui engagent leur travail (COUTROT, 2017). Mais

\footnotetext{
${ }^{1}$ Professeur émérite de psychologie du travail au CNAM, Paris-FR, yves.clot@lecnam.net
} 
quand ce n'est pas le cas - ce qui est très fréquent - il semble qu'il vaille mieux être informé mais pas consulté si l'on n'est pas ensuite effectivement entendu: $13 \%$ des personnes consultées mais finalement pas écoutées ont un symptôme dépressif, soit trois points de plus que celles qui disent avoir été bien informées mais pas vraiment consultées. Être consulté sans pouvoir influer sur les décisions est donc pire que tout. On ne saurait mieux dire la portée du pouvoir d'agir effectif en matière de santé: sans pouvoir changer quoi que ce soit dans une situation mieux vaut se taire. La parole inutile complète régulièrement le tableau du "travail ni fait ni à faire".

\section{Quelques exemples}

Prenons quelques exemples des conséquences de ce sacrifice du "travail bien fait" sur la santé publique. Le premier est devenu un cas d'école: chez Volkswagen l'affaire de la fraude aux émissions de dioxyde de carbone et de gaz polluants a plongé le groupe automobile dans la pire crise de son histoire. La fraude des moteurs Diesel concernait non seulement les émissions d'oxyde d'azote, mais des rejets de $\mathrm{CO} 2$ dans l'atmosphère touchant 11 millions de moteurs (CLOT, 2016; AGGERI; SAUSSOIS, 2017). Cette fraude n'était pas un secret chez Volkswagen comme l’a révélé la presse allemande dès janvier 2015. Un cercle élargi de salariés aurait évoqué dès novembre 2006 l'utilisation d'un logiciel trompeur dans les moteurs pour passer les tests aux émissions. Au sein du département concerné du groupe, la fraude est maintenant considérée comme un acte de désespoir des développeurs, ingénieurs et techniciens, ayant fait "vœu de silence" en sachant qu'il était impossible de respecter les limites officielles d'émission imposées par les autorités de contrôle. Mis sous une énorme pression par leurs dirigeants pour trouver, d'abord pour le marché américain, un système rapide et peu coûteux permettant de rendre les moteurs Diesel "propres", ils n'ont pas osé avouer à la direction qu'aucune solution technique légale n'était envisageable dans les délais imposés. Pris au piège de ce conflit de critères entre qualité et délais, paniqués à l'idée de devoir demander une rallonge de moyens pour développer un nouveau système d'échappement ils se sont tus. Pour les dirigeants du groupe, en matière de prouesse technique, rien ne devait alors être impossible. II n'a pas été utile que ces derniers soient même au courant de cette manipulation et encore moins qu'ils soient à l'origine de la 
fraude. Le rapport social dans le travail ordinaire a suffi. Le travail subordonné a fait son œuvre de refoulement.

Les incidences de ce sacrifice du travail bien fait ne sont d'ailleurs pas repérables seulement sur la santé publique. Le "dieselgate" et les logiciels truqués chez Volkswagen ont, par exemple, emporté deux présidents du Groupe (Ferdinand Piëch et Matthias Müller), conduits le patron d'Audi (Rupert Stadler) en détention provisoire, réduit en fumée 50 milliards d'euros de valeur boursière et coûté pour le moment au Groupe 28 milliards de frais de justice, amendes et indemnisations diverses. Et ce n'est là qu'un exemple de ces "vengeances" du réel quand les conflits de critères n'ont pas droit de cité dans l'organisation, quand aucune institution ne permet leur instruction dialogique (CLOT, 2019a). Atteintes à la santé au travail et risque en matière de santé publique se marient alors avec un gâchis de la performance, même financière.

Prenons un deuxième exemple récent. La construction de I'EPR de Flamanville a débuté en 2007 pour un lancement prévu initialement en 2012, et devait coûter 3 milliards d'euros. Mais les retards n'ont cessé de s'enchaîner, tandis que la facture s'est considérablement alourdie. Le chargement du combustible est désormais prévu en 2021 et l'objectif de coût de construction est porté à 10,9 milliards d'euros. Bientôt huit années de retard et plus de trois fois le prix. Or l'explication d'un tel gaspillage d'énergie, d'efforts et d'argent est venu des sommets mêmes de I'Etat. Elle est contenue dans le rapport de J.M. Folz commandité par le gouvernement et remis tout récemment à la direction d'EDF en Octobre 2019. Sa conclusion est au cœur de la question puisqu'elle met en rapport l'exigence de sécurité nucléaire et l'organisation du travail. Le diagnostic, pourtant retenu, est mordant: "La multiplication, pourtant avérée, des contrôles à tous les niveaux ne suffisait pas à garantir la qualité des réalisations. C'est pourtant bien cette exigence de qualité à toutes les étapes du processus, depuis les études de conception jusqu'aux spécifications techniques de détail, depuis les opérations lourdes de fabrication jusqu'au dernier geste du soudeur, qui fera le succès des chantiers futurs" (FOLZ, 2019, p.32). Du coup, "le développement ou le renouveau d'une culture de qualité, vécue tant comme une obligation collective qu'une ambition individuelle" (FOLZ, 2019, p.32-33) est donnée dans le rapport comme une alternative au sacrifice de la "conscience professionnelle" constatée à plusieurs niveaux.

Les exemples analysés sont nombreux. L'Agence de Sureté Nucléaire avait d'ailleurs alerté sur ce genre de détails qui n'en sont pas, “une vanne montée à l'envers", par exemple ou 
encore l'augmentation de $140 \%$ de la longueur des tuyauteries nécessaires sur un contrat à la suite des très nombreuses modifications sur le chantier imputables à des bureaux d'études s'étant dans plusieurs cas "coupés des réalités du monde industriel en émettant des spécifications irréalisables ou en tombant dans les excès de l'over-engineering" (FOLZ, 2019, p.28). Mais c'est peut-être l'histoire du couvercle de la cuve du réacteur de l'EPR qui donne la meilleure illustration des vicissitudes de la fabrication industrielle puisque, en Novembre 2010, AREVA, en charge du chantier, y détecte des défauts sur $80 \%$ des soudures faites à l'usine de Chalon St Marcel. Et ce n'est qu'en 2018 que sera réalisée l'épreuve hydraulique complète de la cuve avec son couvercle. La piètre qualité du travail de soudure aura été au bout du compte le chemin de croix d'EDF sur ce chantier? sans que les forces de rappel du professionnalisme n'aient pu jouer leur rôle.

Deux autres exemples, parmi d'autres: I'usine de SANOFI de Mourenx qu'il a finalement fallu fermer. Les rejets toxiques de l'usine qui fabriquait la Dépakine, ce médicament contre l'épilepsie dangereux pour les femmes enceintes et leurs foetus, atteignaient, au printemps 2018, date des dernières mesures disponibles, des niveaux astronomiques: jusqu'à 190000 fois au-dessus du plafond autorisé pour le bromopropane, une substance pouvant nuire a la fertilité et au foetus. Le chiffre parait démesuré, impossible a croire. Mais les atteintes à la santé publique se marient avec celles de la santé au travail: onze salariés de l'usine présentent alors des traces de valproate de sodium dans leur sang, une substance cancérogène, mutagène ou toxique pour la reproduction, alors qu'ils ne prennent pas ce médicament.

\section{Agir avec qui?}

Finissons par Lactalis pour réfléchir sur les perspectives d'action. Car, dans ce dernier cas - rappelons qu'il s'agit du lait infantile Lactalis contaminé aux salmonelles en décembre 2017 sans doute en raison d'un défaut d'hygiène autour d'un silo pendant des travaux - l'une des leçons tirées, alors même que le travail dans l'entreprise est questionné par un rapport parlementaire (HUTIN; BESSON-MOREAU, 2018) est la suivante: la répression des fraudes a mené des expériences pour permettre aux clients de signaler en ligne tout problème sur un article. Transformer les consommateurs en lanceurs d'alerte pour détecter plus rapidement des produits 
dangereux est l'un des volets du chantier d'amélioration des procédures de retrait-rappel lancé à la suite de l'affaire. Ce scandale sanitaire avait fait éclater au grand jour des dysfonctionnements sur toute la chaîne de procédures officielles de contrôle. Le gouvernement avait alors décidé, en concertation avec les associations de consommateurs, de mettre en place un système d'information avec un site Internet unique et une application sur mobile.

On ne peut que se féliciter des efforts d'analyse réalisés par les nombreuses commissions d'enquête parlementaire dans ce genre de situations. Et on ne peut sûrement pas sous-estimer non plus l'entrée potentielle des consommateurs et des usagers dans la boucle de contrôle et de décision sur la qualité des produits. Mais une autre question se pose et se posera de plus en plus: dans les entreprises elles-mêmes quel peut-être le rôle en la matière de ceux qui travaillent sans pouvoir se reconnaître dans ce qu'ils font et qui sont bien placés pour alerter en temps utile sur la dégradation de la qualité du travail? Permettre aux clients de signaler les transgressions est sans doute indispensable. Mais permettre aux professionnels de la fabrication d'alerter sur elles ne l'est pas moins. Ce droit est pourtant un impensé de beaucoup d'analyses. Dans tous ces exemples, des conflits de critères dans les fabrications elles-mêmes ont, sans aucun doute possible, affecté et ré-affecté la définition de la qualité du travail. Et chaque fois on mesure l'absurdité où peut conduire la responsabilité exclusive de l'employeur dans la détermination discrétionnaire des critères du travail de qualité. Ce privilège de l'employeur de décider seul de l'objet de l'activité, de son contenu et des critères de sa qualité, surtout lorsque ce privilège cède à la tentation monologique, est de plus en plus hors de saison au regard des risques de santé publique. Ceux qui sont en première ligne du "travail ni fait ni à faire" ne pourront pas durablement être effacés d'une "coopération conflictuelle" toujours plus nécessaire autour de la qualité du travail car ils constituent une force de rappel vitale.

\section{Instituer le conflit de critères pour rendre justice à l'activité}

Au moment où le droit du travail et les institutions représentatives du personnel sont bousculés par des réformes profondes et discutables (SACH; WOLMARK, 2017) on se refuse encore à rendre justice à l'activité réelle de ceux qui travaillent, à leur jugement - bien sûr luimême discutable - à la dignité de leur expérience sensible. Pourtant seul un "professionnalisme 
délibéré" peut protéger la santé publique sous l'autorité d'un travail soigné où progresse l'éthique du travail collectif (CLOT, 2020). Or, les conflits de critères autour du "travail bien fait" n'ont toujours pas d'institutions qui puissent pratiquer les "disputes de métier" vitales pour la santé. Et ce, alors même que l'expérience prouve que ces controverses préventives au travail rendent visibles les incertitudes, impose l'examen les pistes écartées et inventent collectivement des solutions auxquelles personne n'avait pensé auparavant (BONNEFOND, 2019; MIOSSEC; SIMONET, 2019). Les dispositifs de controverses professionnelles sont des institutions qui permettent d'entrer de plein pied dans l'instruction des dossiers préparés par les professionnels eux-mêmes soumis, pour parler comme les juristes, "au principe du contradictoire" dans l'analyse de leur travail. Dans l'apprentissage fait alors ensemble des mondes concrets du travail possible, ce qui progresse c'est l'exploration des dangers en même temps que l'expérience des moyens de les conjurer. Ces "disputes sur la qualité du travail" sont praticables dès aujourd'hui et les conflits de critères institués peuvent devenir la source même de l'intelligence et de la création collective. La santé psychique au travail en sort grandie (BONNEFOND, 2019; BONNEMAIN, 2020; MIOSSEC; SIMONET, 2019; ZITTOUN; LARCHEVÊQUE, 2018).

Toutes les expériences existantes montrent que le pouvoir d'agir contre la qualité empêchée (CLOT, 2008) force le respect et fait autorité dans l'organisation. Mais c'est au prix du recul de la subordination au principe même du droit du travail actuel. C'est en prenant des libertés avec le droit d'aujourd'hui qu'on prépare peut-être le droit du travail de demain (CLOT, 2019b; CLOT; GOLLAC, 2014; WOLMARK, 2016), celui qui devrait inscrire jusque dans le contrat de travail lui-même le droit de contrôle individuel et collectif des professionnels sur leur activité. Contre tout monopole de la décision, l'organisation du travail doit pouvoir faire justice à cette activité au nom de la santé publique. On mesure bien alors que l'Entreprise n'est pas encore I'institution qu'elle doit devenir pour se transformer en opérateur de santé.

Mais on voudrait insister aussi sur la responsabilité à laquelle l'action en ce sens confronte les travailleurs eux-mêmes et les organisations qui veulent les représenter. Pour le faire sans détour, on conclura les quelques remarques qui précèdent en empruntant à Simone Weil le tranchant qu'on lui connaît. On sait que, pour elle, le travail avait quelque chose de sacré: 
Avilir le travail est un sacrilège... Si ceux qui travaillent le sentaient, s'ils sentaient que du fait qu'ils en sont les victimes ils en sont en un sens les complices, leur résistance aurait un tout autre élan que celui que peut leur fournir la pensée de leur personne et de leur droit. Ce ne serait pas une revendication; ce serait un soulèvement de l'être tout entier... Ce sentiment habite bien en eux, mais tellement inarticulé qu'il est indiscernable pour euxmêmes. Les professionnels de la parole sont bien incapables de leur en fournir l'expression. Quand on leur parle de leur propre sort on choisit généralement de leur parler de salaires. Eux sous la fatigue qui les accable et fait de tout effort d'attention une douleur, accueillent avec soulagement la clarté facile des chiffres. Ils oublient ainsi que l'objet à l'égard duquel il y a ainsi marchandage, dont ils se plaignent qu'on les force à le livrer au rabais, qu'on leur en refuse le juste prix, ce n'est pas autre chose que leur âme. Imaginons que le diable est en train d'acheter l'âme d'un malheureux, et que quelqu'un, prenant pitié du malheureux intervienne dans le débat et dise au diable: 'il est honteux de votre part de n'offrir que ce prix; l'objet vaut au moins le double '. Cette farce sinistre est celle qu'a jouée le mouvement ouvrier, avec ses syndicats, ses partis, ses intellectuels de gauche (WEIL, 2018, p.30-31).

On ne peut que se sentir concerné par cette interpellation. Sans doute est-elle sans nuance. Mais, datée de 1950, elle précède pourtant l'installation durable d'un système formel de relations professionnelles dans lequel employeurs et salariés n'ont pu éviter une certaine "corruption" industrielle qui a fait perdre leurs lettres de "noblesse" aux produits, aux objets et maintenant aux services, ne serait-ce que par leur obsolescence programmée. L'innovation ellemême - tellement réelle et fascinante - s'est trouvée pénétrée des passions et des fièvres du consumérisme quand le productivisme a fini par dominer. Cette sorte de déracinement a poussé B. Trentin - cette grande voix du syndicalisme européen en écho à celle de S. Weil -, à faire du travail autre chose qu'une malédiction à dédommager: un nouveau bien public à entretenir ensemble (CLOT, 2019c). Pour ce faire, le barycentre du syndicalisme doit, selon lui, se déplacer en dehors des schèmes de la redistribution et de l'indemnisation, loin d'une pratique de la réparation ou de la compensation juridique ou monétaire de l'oppression contenue dans le travail subordonné (TRENTIN, 2012; 2016). Et ce pour garantir aux travailleurs le droit d'agir, même sans exclusive, sur le travail dans la Cité. Au point où nous en sommes, c'est effectivement la conquête pratique de ce droit inaliénable qui est sacré pour la santé publique. 


\section{Bibliographie}

AGGERI, F.; SAUSSOIS, J. M. De l'affaire Volkswagen au dieselgate: la puissance des grandes entreprises mondialisées à l'épreuve du judiciaire. Revue Française de Gestion, v.269, n.8, p.83100, 2017.

HUTIN, C.; BESSON-MOREAU, G. Tirer les enseignements de l'affaire Lactalis: rapport fait au nom de la commission d'enquête de l'Assemblée nationale. Paris: Assemblée Nationale, 2018.

BONNEFOND, J. Y. L'expérience de Renault Flins: agir sur la qualité du travail. Toulouse: Erès, 2019.

BONNEMAIN, A. Le cas du service de la propreté d'une grande collectivité: intervenir en clinique de l'activité dans l'organisation. Psychologie du Travail et des Organisations. n.27, p.71-85, 2020.

CLOT, Y. Travail et pouvoir d'agir. Paris: Presses Universitaires de France. 2008.

CLOT, Y. Le travail à cœur: Pour en finir avec les risques psychosociaux. Paris: La Découverte, 2010.

CLOT, Y. Clinique, travail et politique. Travailler. n.36, p.91-106, 2016.

CLOT, Y. Préface. In: DETCHESSAHAR, M. (coord.). L'entreprise délibérée: dialoguer pour faire autorité. Paris: Nouvelle Cité, 2019a, p.7-19.

CLOT, Y. Prendre ses responsabilités? De la santé au droit. Sociologie du Travail, v.61, n.2, abr./jun. 2019b, s.p.

CLOT, Y. Le travail: on objet politique sans sujet? In: SUPIOT, A. (coord.) Mondialisation ou globalisation? Les leçons de Simone Weil. Paris: Editions du Collège de France, 2019c, p.131145.

CLOT, Y. Controverses: ethique et travail collectif. Toulouse: Erès. 2020.

CLOT, Y.; GOLLAC, M. Le travail peut-il devenir supportable? Paris: Armand Colin. 2014.

COUTROT, T. Changements organisationnels: la participation des salariés protège-t-elle du risque dépressif?. DARES Analyses. n.061, p.12-15, 2017.

COUTROT, T.; DAVIE, E. Les conditions de travail des salariés dans le secteur privé et la fonction publique. DARES Analyses. n.102, p.1-7, 2014. 
FOLZ, J. M. La construction de l'EPR de Flamanville: rapport au Président Directeur Général d'EDF. 2019.

MIOSSEC, Y.; SIMONET, P. Le 'travail sur le travail' pour agir en santé au travail. In: BERNAUD, J. L.; DESRUMAUX, P.; JEOFFRION, C. (coords.) 10 études de cas en psychologie du travail. Paris: Dunod, 2019, p.133-157.

SACHS, T.; WOLMARK, C. Les réformes 2017: quels principes de composition? Droit Social, n.12, p.1008, 2017.

TRENTIN, B. La Cité du travail. Paris: Fayard. 2012.

TRENTIN, B. Le travail et la liberté. Paris: Editions Sociales. 2016.

WEIL, S. La personne et le sacré. Paris: Editions Allia, 2018.

WOLMARK, C. Le travail absent du droit du travail? Travailler, n.36, p.155-173, 2016.

ZITTOUN, M.; LARCHEVÊQUE, D. Une expérimentation sociale en clinique de l'activité. Rhizome, n.67, p.82-92, 2018.

Recebido em setembro 2020.

Aprovado em setembro 2020. 